\title{
Relação entre os fatores de influência no projeto e a estrutura funcional de uma descascadora de mandioca
}

\author{
Relationship between the design influencing factors and the functional structure \\ of a cassava peeling machine
}

\author{
Ricardo Ferreira Severo ${ }^{\mathrm{I}}$ Leonardo Nabaes Romano ${ }^{\mathrm{II}}$ André Rogério Kinalski Bender $^{\mathrm{III}}$ \\ Natalia Madalena Boelter ${ }^{\text {II }}$
}

\section{RESUMO}

Tipicamente, a mandioca é comercializada na forma in natura, o que requer o descasque manual para posterior consumo humano. Atualmente, é crescente a comercialização da mandioca sem casca, pré-cozida e congelada, no entanto, o beneficiamento é realizado de forma artesanal. Este artigo trata do estudo da operação de retirada da casca e da entrecasca da mandioca, assim como dos demais fatores envolvidos, necessários à construção da base de conhecimentos requeridos para a definição conceitual da estrutura funcional, que expressa um equipamento a ser desenvolvido, destinado a este fim. Assim, o trabalho se delimita às fases iniciais do processo de projeto de produtos industriais. Nesse contexto, o objetivo é verificar se existe relação entre os fatores de influência na operação de descasque e a estrutura funcional de uma descascadora de mandioca. A metodologia empregada baseiase no modelo de referência para o processo de desenvolvimento de máquinas agrícolas, com foco nas atividades concernentes às fases de projeto informacional e conceitual. Os resultados obtidos definem que a análise da operação de descasque, o conhecimento do sistema de cultivo e do processo operacional, as interferências e limites, os requisitos energéticos, o clima, ambiente e a planta são fatores de influência determinantes para a definição completa da estrutura funcional do equipamento. A função global "retirar a casca e a entrecasca da mandioca" se desdobra em quatro funções parciais, as quais decompõem-se em treze funções elementares. Destas, dez funções derivam-se diretamente dos fatores de influência identificados inicialmente. Com isso, verifica-se a existência de relação entre os fatores de influência no projeto e a estrutura funcional do descascador de mandioca, demonstrando dependência entre as definições feitas no início da fase de projeto informacional e as atividades da fase de projeto conceitual, responsáveis pela definição linguística do funcionamento do equipamento e de suas interdependências.

Palavras-chave: agricultura familiar, especificações de projeto, estrutura de funções, projeto de máquinas, processo de projeto.

\section{ABSTRACT}

Cassava is marketed in fresh form, which requires manual peeling for later human consumption. Currently, there is a growing commercialization of peeled cassava, however the processing is done by hand. This article presents the study of the operation to remove the external shell and the inner shell of cassava, as well as others factors involved, necessary to build the knowledge base required for the conceptual definition of functional structure of a cassava peeling machine. This research is delimited to the early stages of the design of industrial products. The objective is to check whether there is a relationship between factors of influence in the operation of peeling and the functional structure of a cassava peeling machine. The methodology is based on a reference model for agricultural machinery development process, with a focus on activities pertaining to the phases of informational and conceptual design. The results establish that the analysis of the operation of peeling are determinants influencing factors for the complete definition of the functional structure. The global function "to remove the external shell and inner shell of cassava" consists in four partial functions that divide into thirteen elementary functions. Of these, ten functions are derived from the influence of the factors initially identified. Thus, it is possible to verify the existence of a relationship between the design influence factors and the functional structure of cassava peeling machine, demonstrating the dependence of the settings made in the informational design phase and the tasks established during the conceptual design phase.

Key words: family farming, design specifications, function structure, machine design, design process.

\section{INTRODUÇÃO}

A mandioca constitui um dos principais alimentos energéticos, sobretudo nos países

\footnotetext{
${ }^{\mathrm{I}}$ In memoriam.
}

IDepartamento de Engenharia Mecânica, Centro de Tecnologia (CT), Universidade Federal de Santa Maria (UFSM), Av. Roraima, 1000, Prédio 07, Sala 312, 97105-900, Santa Maria, RS, Brasil. E-mail: romano@mecanica.ufsm.br.*Autor para correspondência.

IIIPrograma de Pós-graduação em Engenharia Agrícola (PPGEA), Centro de Ciências Rurais (CCR), UFSM, Santa Maria, RS, Brasil. Recebido 22.10.12 Aprovado 26.02.14 Devolvido pelo autor 01.07.14 
em desenvolvimento. No entanto, é cultivada e processada (descascada) com baixo nível tecnológico e em pequenas propriedades, geralmente de agricultura familiar (FUKUDA \& OTSUBO, 2003). No descascamento, os cuidados com a higiene e a segurança são fundamentais, a fim de evitar que as bactérias iniciem seu processo de proliferação, além da atenção, pois o trabalho é realizado com o uso de facas (SEVERO, 2012). Com o consumo crescente da mandioca sem casca (pré-cozida e congelada), os trabalhadores podem se beneficiar com a mecanização da operação de descasque, tanto em termos de aumento de produtividade, como em melhorias na segurança do trabalhador. No entanto, para que isso ocorra, requer-se o desenvolvimento de ações tecnológicas de pesquisa que produzam soluções mecanizadas para a operação de descasque. Estas, quando obtidas, testadas e aprovadas poderão ser produzidas industrialmente e comercializadas, caracterizando, portanto, uma importante inovação para a agricultura familiar, que é carente de tecnologias de auxílio ao trabalho do homem no campo.

Apesar do consumo de mandioca de mesa ser elevado, sua produção real é desconhecida, pois grande parte ocorre por produção caseira e não passa na sua totalidade por um processo formal de comercialização (BORGES et al., 2002). Estas empresas são indústrias domésticas rurais (OLIVEIRA, 2010) e representam um bom exemplo de atividade da agricultura familiar, a qual deve ser viabilizada, pois gera mais ocupações do que a agricultura patronal, podendo utilizar de forma eficiente os recursos terra, trabalho e capital, além de irradiar maior desenvolvimento local (GUANZIROLI et al., 2001).

Uma vez caracterizada a demanda para o desenvolvimento de uma máquina destinada ao descasque de mandioca para o consumo de mesa, esta poderá ser obtida por meio da realização de um processo de projeto sistematizado (BACK et al., 2008). Tipicamente, o processo de projeto de um produto industrial é decomposto em quatro fases fundamentais (BACK et al., 2008): informacional; conceitual; preliminar; e projeto detalhado. As duas primeiras fases são responsáveis, respectivamente, pela determinação das especificações de projeto da máquina agrícola e pela concepção do produto. Já as fases de projeto preliminar e detalhado abrangem o detalhamento do projeto da máquina, a determinação da viabilidade econômica, a construção e os testes para aprovação do protótipo. Assim, se um projeto é empreendido na busca de uma solução conceitual inovadora e duradoura para uma determinada função, faz-se imperioso considerar um conjunto de informações básicas para a condução do projeto (ROMANO et al., 2005). Nesse sentido, MARINI (2007) cita a importância de levantar os fatores que influem na definição das especificações de projeto da máquina ou equipamento. Isso significa conhecer, além dos requisitos de projeto derivados das necessidades dos clientes, as propriedades dos elementos do ciclo de vida que podem influenciar no alcance dos objetivos do projeto.

Do ponto de vista operacional, os fatores de influência no projeto foram estabelecidos, primeiramente, no campo das máquinas agrícolas, como um conjunto de parâmetros que afeta o seu desempenho (CHRISTIANSON \& ROHRBACH, 1986). Entretanto, segundo MARINI \& ROMANO (2009), esta definição necessitava de uma base que melhor especificasse o seu escopo, porque sua forma original se sobrepunha à definição de especificação de projeto, como estabelecida por ULRICH \& EPPINGER (1995), PAHL et al. (2005) e FONSECA (2000).

Os fatores de influência no projeto consistem em propriedades do ciclo de vida dos produtos cuja identificação e análise criam bases seguras (conhecimento específico) para a realização de ações e tomadas de decisão na fase de concepção da máquina agrícola (ROMANO, 2013). É possível afirmar que esse conhecimento, ao ser adequadamente classificado e descrito, serve de referência no reconhecimento de restrições e riscos ao projeto de máquinas agrícolas (MARINI et al., 2006b). Logo, a determinação da modalidade de cultivo, em conjunto com a definição e a análise das operações agrícolas que devem ser executadas é o ponto de partida fundamental para a definição das funções necessárias ao projeto da máquina, de modo a satisfazer as necessidades de seus clientes de utilização, levando-se em conta a conformação da análise da operação agrícola (MARINI et al., 2006a). Exemplos de análise da operação podem ser vistos em ALBIERO et al. (2011) e ALBIERO et al. (2012).

Ao longo das duas primeiras fases do processo de projeto, MARINI \& ROMANO (2007) preconizam uma relação direta entre os fatores de influência no projeto e o estabelecimento da estrutura funcional de uma máquina agrícola. A fim de comprovar esta afirmação, o objetivo do trabalho é verificar se existe relação entre os fatores de influência na operação de descasque e a estrutura funcional de uma descascadora de mandioca. Conforme MARINI (2007), o conhecimento do conceito funcional é importante para configurar as operações a serem executadas pelos conjuntos mecânicos, em adequação às necessidades dos usuários. 


\section{MATERIAL E MÉTODOS}

A metodologia utilizada para estabelecer a existência de relação entre os fatores de influência no projeto e a estrutura funcional de uma máquina de descascar mandioca tem como base o Modelo de Referência para o Processo de Desenvolvimento de Máquinas Agrícolas (MR-PDMA) de ROMANO (2013) e o método de determinação dos fatores de influência no projeto de MARINI (2007). Tal abordagem pode ser classificada como um estudo de caso, pela utilização de uma circunstância real da atividade de projeto. Assim, para os propósitos deste artigo, a pesquisa foi dividida em três etapas.

A primeira, parte da fase de projeto informacional (atividade de definição dos fatores de influência no projeto), consistiu na busca de dados e informações pela abordagem de pesquisa exploratória, com o intuito de obter conhecimento sistemático e lógico dos parâmetros que norteiam a caracterização da operação agrícola "descascar mandioca”. O conjunto de dados e informações de interesse ao projeto são transformados em fatores de influência e classificados em classes, propriedades, informações e descrição. As classes trabalhadas referem-se: (i) ao sistema de cultivo; (ii) ao processo operacional; (iii) interferências e limites; (iv) requisitos energéticos; (v) clima e ambiente; (vi) planta; e (vii) análise da operação. Para a organização dos dados coletados, foram utilizadas planilhas eletrônicas, baseadas no modelo proposto por MARINI (2007), cujos resultados estão descritos na seção seguinte. Na segunda etapa, utilizaram-se as informações coletadas em uma prática de projeto da fase de projeto conceitual (atividade de definição da estrutura funcional da máquina), como proposto no MR-PDMA (ROMANO, 2013), de modo a testar a validade das informações obtidas por meio dos fatores de influência no projeto. Na terceira etapa, realizou-se a identificação de que fatores de influência no projeto se relacionam com as funções parciais e funções elementares definidas na estrutura funcional.

\section{RESULTADOS E DISCUSSÃO}

Os fatores de influência relativos à classe "sistema de cultivo" indicam que a principal característica encontrada é a forma da mandioca. Este fator está relacionado com as características do solo e a forma como o plantio é realizado. Outra característica é o desenvolvimento das plantas nas diferentes regiões do país, variando de acordo com a temperatura a qual ela é submetida.
Com relação à classe "processo operacional”, o descasque manual, com ferramenta cortante (faca), resulta na justificativa para a mecanização da operação, uma vez que este processamento mínimo da mandioca apresenta riscos à integridade física dos produtores, apesar de proporcionar uma agregação de valor significativa ao produto.

A classe "interferências e limites" revela que, como se trata de um alimento, o produto a ser processado pela máquina, os cuidados para a preservação da mandioca estão entre os principais fatores que devem ser observados durante o projeto.

A classe "requisitos energéticos" prevê que energia deva ser aplicada aos elementos funcionais (orgãos ativos portadores do efeito físico), responsáveis pela sequência de operações a ser realizada pela máquina.

Os fatores de influência no projeto referente à classe "clima e ambiente" são resultantes de características do clima e não interferem no funcionamento da máquina, mas sim na fisiologia vegetal da mandioca, e esta sim influencia na operação de descasque. Com esse conhecimento, é possível determinar em quais locais e épocas do ano a máquina pode ser utilizada.

A classe de fatores de influência no projeto "planta” caracteriza que a mandioca produz raízes de forma e dimensões variadas, resultando em um produto a ser descascado desuniforme, influenciando diretamente a concepção do mecanismo portador do efeito físico de descasque.

A última classe de fatores de influência analisada refere-se à “operação agrícola” que a máquina deve executar. Pode-se observar que a máquina deve processar a raiz de mandioca identificando, portanto, uma condição de entrada à máquina - que é a mandioca com casca (elemento processado) - e duas condições de saída, a primeira, a mandioca sem casca (elemento resultante 1) e a segunda, a casca (elemento resultante 2). Verificase também a ocorrência de operações auxiliares de armazenamento da mandioca a ser descascada, de transporte, de controle de vazão, de direcionamento da mandioca descascada e da casca, assim como de armazenamento da mandioca descascada e da casca que foi retirada. Estas operações envolvem ações mecânicas e ações de controle, de modo a permitir o processo de beneficiamento da mandioca.

Como preconizado por MARINI et al. (2006b), a identificação, classificação e descrição adequada dos fatores de influência acima apresentados serve de referência no reconhecimento de restrições e riscos ao projeto da máquina descascadora de mandioca, assim como constitui-se em uma base 
segura para a tomada de decisões ao longo do processo de projeto da máquina (CHRISTIANSON \& ROHRBACH, 1986), especialmente no que se refere à definição da estrutura funcional (ROMANO, 2003; ROMANO et al., 2005; MARINI, 2007; MARINI \& ROMANO, 2009). Considerações similares, envolvendo projeto de outros tipos de máquinas agrícolas, também são afirmadas por FLORES et al. (2006), NIETIEDT et al. (2010), BISOGNIN et al. (2010) e BELLÉ et al. (2012).

A partir do conhecimento dos fatores de influência no projeto, foi definida a estrutura funcional da máquina em termos das funções elementares para que a mandioca com casca transforme-se em mandioca sem casca (execução da função global). A estrutura funcional define o primeiro conceito da máquina propriamente dita. Para tal, define-se primeiramente a função global da máquina (nível 1) à luz dos fatores de influência no projeto. A função global, extrair ou retirar a casca e a entrecasca da mandioca, é ilustrada na figura 1 e transforma a entrada de material (mandioca com casca), energia (motora) e sinal (operação não realizada) em saída de material (mandioca descascada e casca), energia (perdida) e sinal (operação realizada). Esta função é por sua vez decomposta em funções menos complexas, denominadas de funções parciais (funções de nível 2, definidas como FP1, FP2, FP3 e FP4) e estas em funções elementares (nível 3, identificadas como FE1.1, FE1.2, FE2.1, FE2.2, FE2.3, FE2.4, FE2.5, FE2.6, FE2.7, FE3.1, FE3.2, FE4.1 e FE4.2). $\mathrm{O}$ diagrama de blocos das funções elementares acima

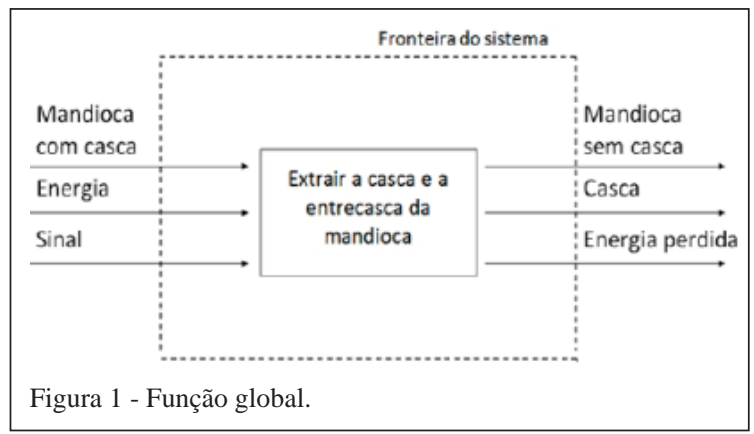

citadas define a estrutura funcional (Figura 2) da descascadora de mandioca.

Assim, a estrutura funcional foi estabelecida de acordo com os preceitos citados por MARINI et al. (2006a), sendo concordante em termos de identificar uma relação direta entre os fatores de influência no projeto e as funções elementares da máquina, ao longo das duas primeiras fases do processo de projeto, como preconizado em MARINI \& ROMANO (2007). Sustenta-se como verdadeira a afirmação de MARINI (2007) de que o conhecimento do conceito funcional é essencial para configurar as tarefas executadas pelos conjuntos mecânicos, em adequação às necessidades dos usuários. Logo, para que as características do ambiente de operação de uma máquina agrícola sejam entendidas completamente, é necessária a tradução destas em informações claras e objetivas e que possam ser interpretadas pelos projetistas, demonstrando a relação entre os fatores de influência no projeto e a estrutura funcional da máquina de descascar mandioca (Tabela 1). Os

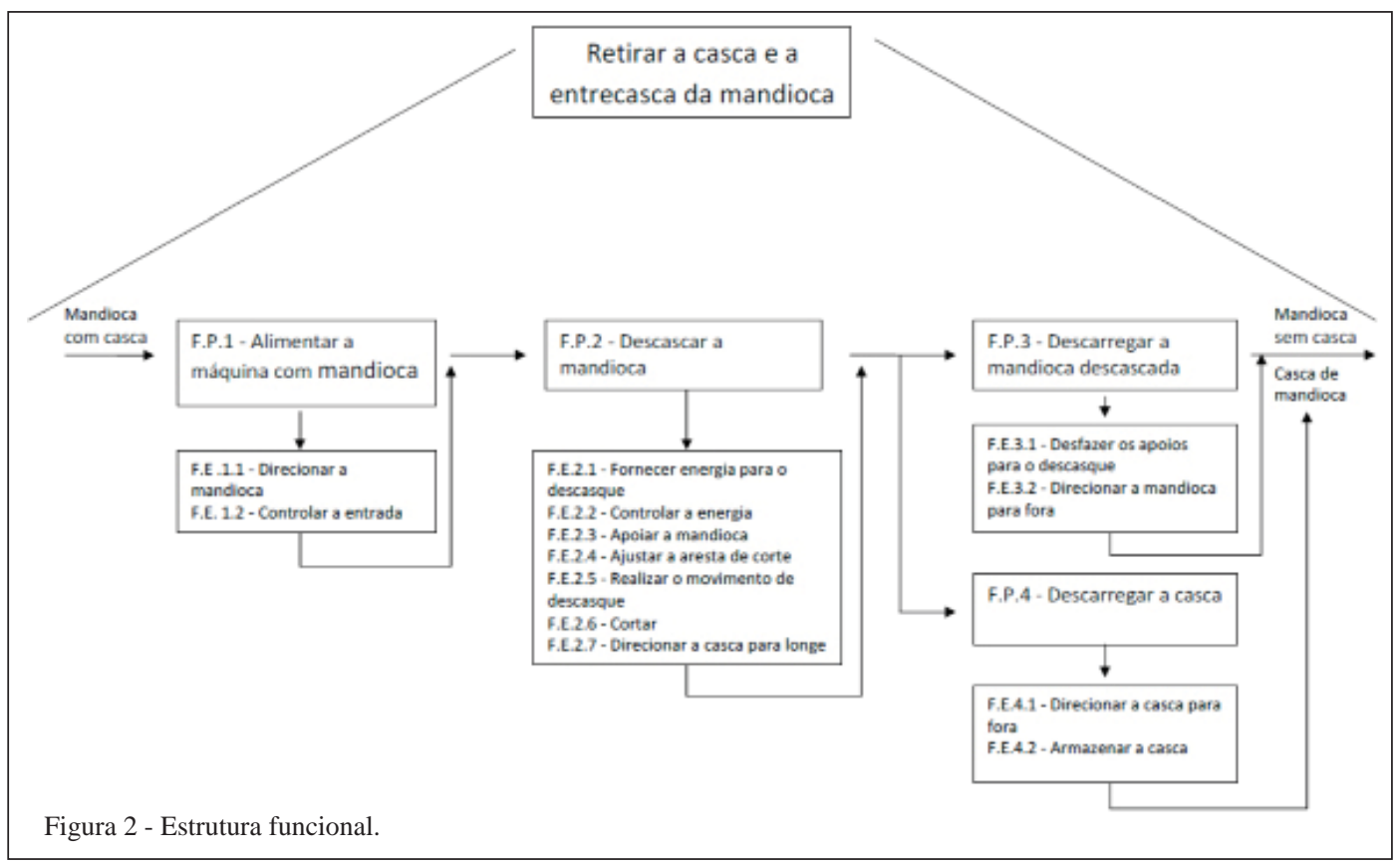

Ciência Rural, v.44, n.9, set, 2014. 
Tabela 1 - Relação entre os fatores de influência e a função elementar da estrutura funcional.

\begin{tabular}{|c|c|c|}
\hline Fator de influência & & Função elementar \\
\hline Interferências e limites & - Alimentar a máquina & F.E.1.1 Direcionar a mandioca \\
\hline Análise da operação & - Dosar a entrada & F.E. 1.2 Controlar a entrada \\
\hline \multirow[t]{2}{*}{ Requisitos energéticos } & $\begin{array}{l}\text { - Utilizar a energia } \\
\text { - Transformar movimentos }\end{array}$ & F.E. 2.1 Fornecer energia para o descasque \\
\hline & - Trabalho não contínuo & $\begin{array}{l}\text { F.E. } 2.2 \text { Controlar a energia } \\
\text { F.E. } 2.3 \text { Apoiar a mandioca }\end{array}$ \\
\hline Planta & - Diâmetro entre 15 e 40mm e comprimento até 400mm & F.E. 2.4 Ajustar a aresta de corte \\
\hline Requisitos energéticos & - Transformar movimentos & F.E. 2.5 Realizar o movimento de descasque \\
\hline Interferências e limites & - Riscar a mandioca & F.E. 2.6 Cortar \\
\hline \multirow{3}{*}{ Análise da operação } & $\begin{array}{l}\text { - Mandioca com casca } \\
\text { - Mandioca sem casca }\end{array}$ & $\begin{array}{l}\text { F.E. } 2.7 \text { Direcionar a casca para longe } \\
\text { F.E. 3.2 Direcionar a mandioca para fora }\end{array}$ \\
\hline & - Casca & F.E. 4.1 Direcionar a casca para fora \\
\hline & - Liberar a mandioca & $\begin{array}{l}\text { F.E. 3.1 Desfazer os apoios para o descasque } \\
\text { F.E. } 4.2 \text { Armazenar a casca }\end{array}$ \\
\hline
\end{tabular}

resultados obtidos apontam que, de um total de treze funções elementares, dez foram definidas tendo como fundamentação técnica os fatores de influência identificados. Esta constatação mostra que o mecanismo para definição do conceito da máquina em questão está coerente com as informações coletadas no início do projeto.

\section{CONCLUSÃO}

Verifica-se a existência de relação entre os fatores de influência na operação de descasque e a estrutura funcional do descascador de mandioca, o que indica uma dependência entre as definições feitas no início da fase de projeto informacional e as atividades da fase de projeto conceitual, responsável pela definição linguística do funcionamento do equipamento, comprovando o preconizado por MARINI \& ROMANO (2007).

\section{REFERÊNCIAS}

ALBIERO, D. et al. Desenvolvimento e projeto de colhedora de babaçu (Orbignya phalerata Mart.) para agricultura familiar nas regiões de matas de transição da Amazônia. Acta Amazonica, v.41, n.1, p.57-68, 2011.

ALBIERO, D. et al. Proposta conceitual de colhedoras autopropelidas de açaí (Euterpe oleracea Mart.) para a Região Amazônica. Revista Ciência Agronômica, v.43, n.2, p.382-389, 2012.

BACK, N. et al. Projeto integrado de produtos - planejamento, concepção e modelagem. Barueri, SP: Manole, 2008. 601p.
BELLÉ, M. P.; et al. Fatores de influência no projeto de máquinas para tratamento de sementes. In: Congresso Brasileiro de Engenharia Agrícola, 41, 2012. Londrina. Anais... Londrina: Associação Brasileira de Engenharia Agrícola, 2012. CD-ROM.

BISOGNIN, A. et al. Fatores de influência no projeto de semeadoras de fluxo contínuo - avaliação de máquinas disponíveis no mercado brasileiro. In: CONGRESSO BRASILEIRO DE ENGENHARIA AGRÍCOLA, 39., 2010, Vitória, ES. Anais... Vitória: Associação Brasileira de Engenharia Agrícola, 2010. CD-ROM.

BORGES. M.F. et al. Avaliação de variedades de mandioca para consumo humano. Pesquisa Agropecuária, v.37, n.11, p.15591565, 2002.

CHRISTIANSON, L.L.; ROHRBACH, R.P. Design in agricultural engineering. St. Joseph: ASABE, 1986. 310p.

FLORES, E.F. et al. Fatores de influência no projeto de uma máquina automotriz para a colheita consorciada de milho (Zea mayz L.) e feijão (Phaseolus vulgaris). In: CONGRESSO BRASILEIRO DE ENGENHARIA AGRÍCOLA, 35., 2006, João Pessoa, PB. Anais... João Pessoa: Associação Brasileira de Engenharia Agrícola, 2006. CD-ROM.

FONSECA, A.J.H. Sistematização do processo de obtenção das especificações de projeto de produtos industriais e sua implementação computacional. p. 180, 2000. Tese (Doutorado em Engenharia Mecânica) - Programa de Pós-graduação em Engenharia Mecânica, Universidade Federal de Santa Catarina, SC.

FUKUDA, C.; OTSUBO, A.A. Cultivo da mandioca na região centro sul do Brasil. EMBRAPA Mandioca e Fruticultura. Brasília: EMBRAPA, 2003. (Sistemas de Produção, 7). Disponível em: <http://sistemasdeproducao.cnptia.embrapa.br>. Acesso em: 12 jan. 2012. ISSN 1678-8796. 
GUANZIROLI, A. et al. Agricultura familiar e reforma agrária no século XXI. Rio de Janeiro: Garamond, 2001. 288p.

MARINI, V.K. Fatores de influência e funções técnicas no projeto de máquinas agrícolas: uma contribuição teórica. 2007. 181f. Dissertação (Mestrado em Engenharia Agrícola) Universidade Federal de Santa Maria, Santa Maria, RS.

MARINI, V.K.; ROMANO, L.N. Influencing factors in agricultural machinery design. Product, v.7, n.2, p.111-130, 2009.

MARINI, V.K.; ROMANO, L.N. The Function Structure as a tool for analyzing an existing concept of a centrifugal fertilizer spreader. Product, v.5, n.2, p.77-90, 2007.

MARINI, V.K. et al. A análise da operação agrícola como base para a definição de requisitos funcionais no processo de desenvolvimento de máquinas agrícolas. In: CONGRESSO BRASILEIRO DE ENGENHARIA AGRÍCOLA, 35., 2006, João Pessoa, PB. Anais... João Pessoa: Associação Brasileira de Engenharia Agrícola, 2006a. CD-ROM.

MARINI, V.K. et al. Considerações fundamentais para a sistematização dos fatores de influência no projeto da máquina agrícola. In: CONGRESSO BRASILEIRO DE ENGENHARIA AGRÍCOLA, 35., 2006, João Pessoa, PB. Anais... João Pessoa : Associação Brasileira de Engenharia Agrícola, 2006b. CD-ROM.

NIETIEDT, G.H. et al. Definição dos fatores de influência no processo de desenvolvimento de uma máquina semeadora adubadora de precisão. In: CONGRESSO BRASILEIRO DE ENGENHARIA AGRÍCOLA, 39., 2010, Vitória, ES. Anais... Vitória: Associação Brasileira de Engenharia Agrícola, 2010. CD-ROM.

OLIVEIRA, A.U. Agricultura e indústria no Brasil. Campo Território: Revista de Geografia Agrária, v.5, n.10, p.5-64, 2010.

PAHL, G. et al. Projeto na engenharia: fundamentos do desenvolvimento eficaz de produtos, métodos e aplicações. São Paulo: Edgard Blücher, 2005. 412p.

ROMANO, L.N. Desenvolvimento de máquinas agrícolas: planejamento, projeto e produção. São Paulo: Blucher Acadêmico, 2013. 310p.

ROMANO, L.N. et al. An introduction to the reference model for the agricultural machinery development process. Product, v.3, n.2, p.109-132, 2005. Disponível em: <http://pmd.hostcentral. com.br/search.php?busca =vol_03/nr_2\#revista>. Acesso em: 10 nov. 2011. ISSN 1676-4056.

SEVERO, R.F. Projeto conceitual e teste do modelo funcional de um descascador de mandioca. 2012. 110f. Dissertação (Mestrado em Engenharia Agrícola) - Universidade Federal de Santa Maria, Santa Maria, RS.

ULRICH, K.T.; EPPINGER, S.D. Product design and development. New York: McGraw-Hill, 1995. 289p. 